\title{
Cavopulmonary assist for the univentricular Fontan circulation: von Kármán viscous impeller pump
}

\author{
Mark D. Rodefeld, MD, ${ }^{\mathrm{a}}$ Brandon Coats, MS, ${ }^{\mathrm{b}}$ Travis Fisher, MS, ${ }^{\mathrm{b}}$ Guruprasad A. Giridharan, PhD, ${ }^{\mathrm{c}}$ \\ Jun Chen, PhD, ${ }^{\mathrm{b}}$ John W. Brown, MD, ${ }^{\mathrm{a}}$ and Steven H. Frankel, $\mathrm{PhD}^{\mathrm{b}}$
}

Objective: In a univentricular Fontan circulation, modest augmentation of existing cavopulmonary pressure head
$(2-5 \mathrm{~mm} \mathrm{Hg}$ ) would reduce systemic venous pressure, increase ventricular filling, and thus substantially improve
circulatory status. An ideal means of providing mechanical cavopulmonary support does not exist. We hypoth-
esized that a viscous impeller pump, based on the von Kármán viscous pump principle, is optimal for this role.

Methods: A 3-dimensional computational model of the total cavopulmonary connection was created. The impeller was represented as a smooth 2 -sided conical actuator disk with rotation in the vena caval axis. Flow was modeled under 3 conditions: (1) passive flow with no disc; (2) passive flow with a nonrotating disk, and (3) induced flow with disc rotation $(0-5 \mathrm{~K} \mathrm{rpm})$. Flow patterns and hydraulic performance were examined for each case. Hydraulic performance for a vaned impeller was assessed by measuring pressure increase and induced flow over 0 to $7 \mathrm{~K}$ rpm in a laboratory mock loop.

Results: A nonrotating actuator disc stabilized cavopulmonary flow, reducing power loss by $88 \%$. Disk rotation (from baseline dynamic flow of $4.4 \mathrm{~L} / \mathrm{min}$ ) resulted in a pressure increase of $0.03 \mathrm{~mm} \mathrm{Hg}$. A further increase in pressure of 5 to $20 \mathrm{~mm} \mathrm{Hg}$ and 0 to $5 \mathrm{~L} / \mathrm{min}$ flow was obtained with a vaned impeller at 0 to $7 \mathrm{~K} \mathrm{rpm}$ in a laboratory mock loop.

Conclusions: A single viscous impeller pump stabilizes and augments cavopulmonary flow in 4 directions, in the desired pressure range, without venous pathway obstruction. A viscous impeller pump applies to the existing staged protocol as a temporary bridge-to-recovery or -transplant in established univentricular Fontan circulations and may enable compressed palliation of single ventricle without the need for intermediary surgical staging or use of a systemic-to-pulmonary arterial shunt. (J Thorac Cardiovasc Surg 2010;140:529-36)

In Fontan repair of functional single ventricle, a staged surgical approach is necessary to achieve the end goal of a series connection of the systemic venous return to the pulmonary arteries. Despite medical and surgical advances, however, this approach remains problematic. ${ }^{1}$ At the expense of providing a reliable source of neonatal pulmonary blood flow, interim use of a systemic-to-pulmonary arterial shunt creates serious pathophysiologic consequences. Shunt physiology may impair timing for Fontan conversion and detrimentally affect late Fontan status. ${ }^{2}$ Once Fontan palliation is complete, the absence of a subpulmonary ventricle results in

From the Section of Cardiothoracic Surgery, ${ }^{\mathrm{a}}$ Department of Surgery, Indiana University School of Medicine and James Whitcomb Riley Hospital for Children, Indianapolis, Ind; The Department of Mechanical Engineering, ${ }^{\mathrm{b}}$ Purdue University, West Lafayette, Ind; and The Department of Bioengineering, ${ }^{c}$ University of Louisville, Louisville, Ky.

This work was supported in part by National Institutes of Health Grant HL080089 (MDR); and Collaboration in Bioengineering Grant (MDR, SHF) and Research Support Funds Grant (MDR), Indiana University Purdue University, Indianapolis. Disclosures: None.

Presented at the 89th Annual Meeting of the American Association for Thoracic Surgery, Boston, Massachusetts, May 6-13, 2009.

Received for publication May 8, 2009; revisions received Nov 19, 2009; accepted for publication April 10, 2010; available ahead of print June 21, 2010.

Address for reprints: Mark D. Rodefeld, MD, Associate Professor of Surgery, Section of Cardiothoracic Surgery, Indiana University School of Medicine, Emerson Hall 215, 545 Barnhill Drive, Indianapolis, IN 46202 (E-mail: rodefeld@iupui.edu). $0022-5223 / \$ 36.00$

Copyright (C) 2010 by The American Association for Thoracic Surgery doi:10.1016/j.jtcvs.2010.04.037 chronic elevation in systemic venous, capillary, and interstitial pressures; reduction in ventricular preload; and subnormal cardiac output for the duration of a patient's life. These problems can be addressed by pump augmentation of existing Fontan cavopulmonary flow. ${ }^{3,4}$

A pump to accomplish this does not currently exist. If a safe and reliable device were developed, it may be possible to stabilize patients with "failing Fontan" physiology, early or late after repair, by temporarily reversing the Fontan paradox of elevated venous pressure and impaired ventricular filling. ${ }^{5}$ We hypothesized that a viscous impeller pump, based on the von Kármán viscous pump principle, possesses qualities that are ideal to serve in this capacity. ${ }^{6,7}$ This report presents an initial analysis, and background and rationale, for a viscous impeller pump to provide cavopulmonary assist.

\section{MATERIALS AND METHODS}

Computational Fluid Dynamic Analysis of an Actuator Disk Within a Total Cavopulmonary

\section{Connection}

Computational fluid dynamic (CFD) (Fluent, Ansys Inc, Canonsburg, $\mathrm{Pa}$ ) analysis was used to examine the hydraulic performance and flow conditions of a viscous impeller pump (tip diameter, $20 \mathrm{~mm}$; hub diameter, 6 $\mathrm{mm}$ ) in an idealized adult total cavopulmonary connection (TCPC) model of semi-physiologic flow (22-mm diameter inferior vena cava [IVC]/superior vena cava [SVC], 19-mm diameter left pulmonary artery/right pulmonary artery, slightly expanded at junction resulting in tip gap clearance of $8 \mathrm{~mm}, 4.4 \mathrm{~L} / \mathrm{min}$ baseline flow, 50/50 inlet-outlet split) (Figure 1). These 


\section{Abbreviations and Acronyms \\ CFD = computational fluid dynamic \\ IVC = inferior vena cava \\ $\mathrm{SVC}=$ superior vena cava \\ $\mathrm{TCPC}=$ total cavopulmonary connection}

are reasonable estimates of adult TCPC dimensions and flow, and provide a reasonable clinical safety margin against impeller strike and vessel injury.

The following conditions were considered: (1) absence of the pump; (2) presence of a stationary pump; and (3) presence of a rotating pump $(0-5000$ rpm). Impeller geometry for the CFD studies was patterned as a smooth surfaced, 2-sided, centrifugal pump. Tetrahedral meshes were produced using Gambit (preprocessor for Fluent) with 900,000 elements. Laminar flow was assumed for the case without the pump and with the stationary pump. The unsteady Reynolds-averaged Navier-Stokes equations using the realizable k- $\epsilon$ turbulence model, no-slip boundary conditions, and $y+$ grid requirements were integrated within Fluent only for the case of the rotating pump. The inlet turbulence intensity was selected as $3 \%$, and variation of this value did not significantly affect the results. The fluid medium was assigned a physiologic density of $1060 \mathrm{~kg} / \mathrm{m}^{3}$ and viscosity of $3.5 \mathrm{cP}$; both were assumed to be constant.

\section{In Vitro Hydraulic Studies}

The hydraulic performance of a vaned impeller was assessed in an in vitro mock circulatory loop with a 4-way TCPC conduit. The mock loop used compliance and resistance elements to mimic the physiologic parameters of an adult Fontan circulation. ${ }^{8}$ The impeller tested was constructed as a stereolithography rapid-prototype from computer-aided design drawings using optically transparent Accura 60 resin (Forecast 3D, Carlsbad, Calif). Impeller and conduit dimensions used in the mock circulatory loop were identical to the CFD model, with the exception that surface vane structure was added to the impeller surface. This adult-scale, rapid-prototype impeller simulates the expanded device and is $18 \mathrm{~mm}$ in diameter and $6 \mathrm{~mm}$ in length. The impeller is intended to be collapsed to $2.86 \mathrm{~mm}$ in diameter to fit an $8 \mathrm{~F}$ catheter. The operational range is 0 to $7000 \mathrm{rpm}$. There are 4 vanes on the version tested, each $2 \mathrm{~mm}$ in height at the center and blended at inflow hub and outflow tip. An external brushless direct-current motor was used to drive the impeller in the Fontan mock circuit.

\section{RESULTS \\ Computational Fluid Dynamic Modeling and Flow Patterns}

A mid-plane contour plot of instantaneous velocity magnitude with streamlines, with and without a stationary pump present, is shown in Figure 2. TCPC flow patterns in the absence of a central stabilizing body (actuator disk/pump) match previously reported studies using CFD and magnetic resonance imaging, where irregular flow in the central stagnation region accounts for energy loss and inefficiency. ${ }^{9,10}$ In the presence of the stationary pump, TCPC flow patterns are improved with $88 \%$ reduction in power loss (Table 1 ). Thus, the presence of the pump, rotating or not, has a marked stabilizing effect in this 4-way flow configuration.

\section{Predicted Hydraulic Performance and Wall Shear Stress}

CFD predictions for hydraulic performance of a smoothsurfaced impeller within the TCPC, and comparison with

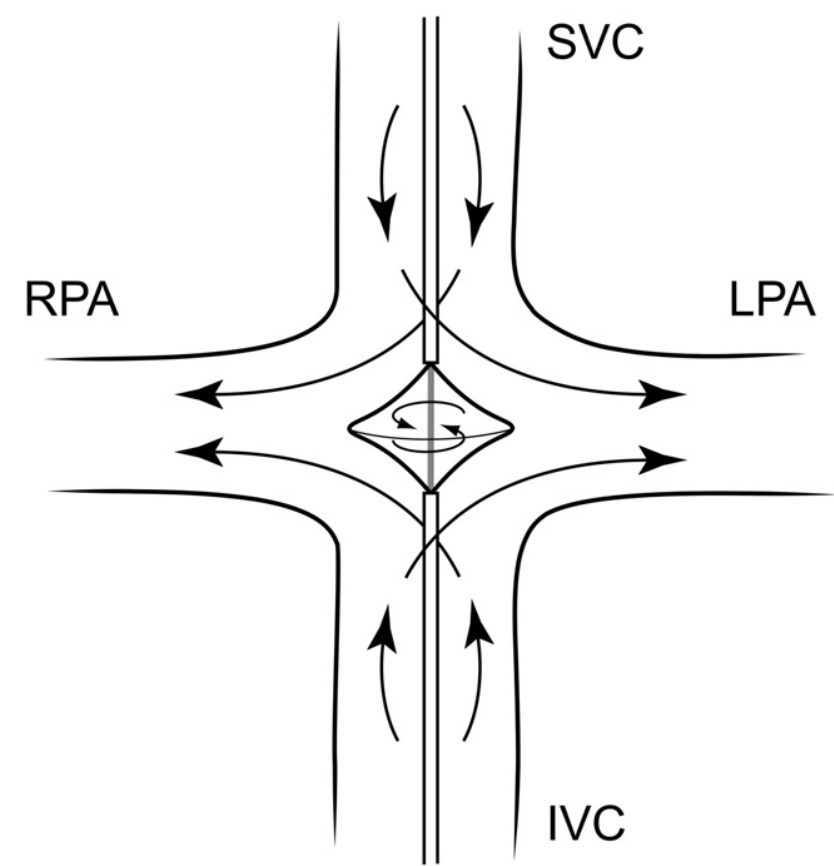

FIGURE 1. Concept of a viscous impeller pump to provide cavopulmonary assist. $S V C$, Superior vena cava; $I V C$, inferior vena cava; $L P A$, left pulmonary artery; $R P A$, right pulmonary artery.

a TCPC without a pump, are shown in Table 1. According to the boundary conditions used as constraints in the computations, the flow rate remained constant for each case $(4.4 \mathrm{~L} /$ $\mathrm{min}$ ), whereas the effect of the pump was demonstrated in generating a pressure increase, thus reducing power loss $(\triangle \mathrm{P}$ $[\mathrm{Pa}] \times 4.4 \mathrm{~L} / \mathrm{min}$ and then converted to $\mathrm{m}^{3} / \mathrm{s}$ ) within the fluid of the model. Although the pressure increase in each case is comparatively small, viscous flow losses are negated by the actuator disk between 1000 and $2500 \mathrm{rpm}$, whereon the function of the disk makes a transition from the role of flow stabilizer to that of a pump. Axial (vena caval) inflow increased consistent with pressure generated by higher rates of impeller rotation.

We further used CFD to identify regions of both high stress (hemolysis and platelet activation) and low stress (flow stasis and thrombogenicity) within the impeller. Scalar stress estimations for the smooth impeller at $5000 \mathrm{rpm}$ are shown in Figure $3, A .{ }^{11}$ Relatively low scalar stress is found on the surface of the pump and rotation of the fluid as it transitions from axial to radial flow. All stress values were greater than zero. Scalar stress values on the impeller surface were less than $100 \mathrm{~Pa}$, with the highest levels at the outflow edge of the impeller (Figure 3, B). Efficiency can be optimized by modifying pump geometry to distribute flow from the pump periphery over the entire cross-sectional area of the left and right pulmonary arteries. As spatial distribution is optimized and magnitude of turbulence is minimized, the risk of shearinduced blood damage is reduced. ${ }^{11-13}$ We expect scalar 


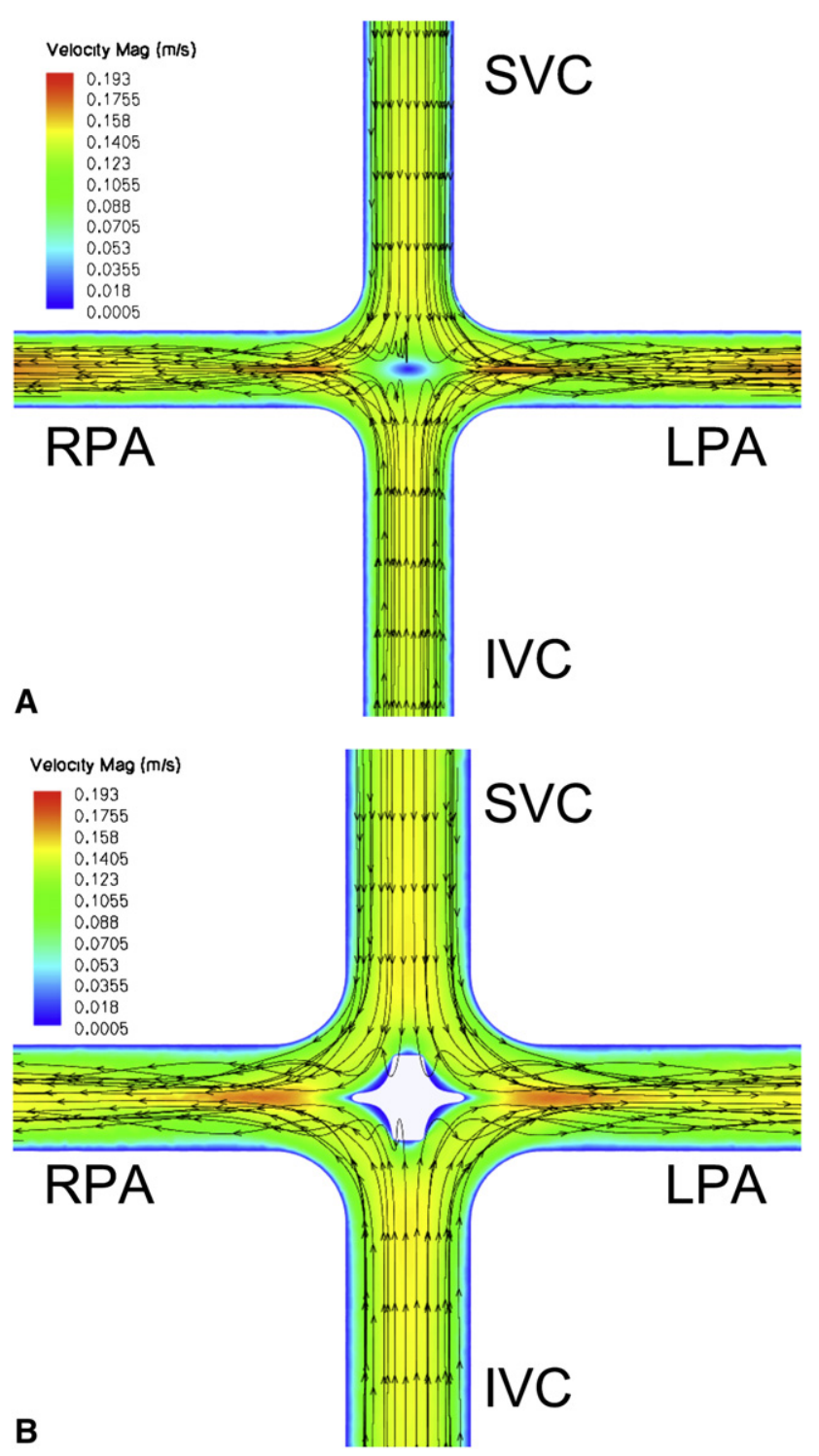

FIGURE 2. Velocity magnitude $(\mathrm{m} / \mathrm{s})$ contour plot within the TCPC at flow of $4.4 \mathrm{~L} / \mathrm{min}$. A, No impeller. Incoming flow is irregular at the intersection. B, Stationary impeller. Flow pattern is stabilized, reducing power loss by $88 \%$. $S V C$, Superior vena cava; $I V C$, inferior vena cava, $L P A$, left pulmonary artery; $R P A$, right pulmonary artery.

stress with the viscous impeller pump will remain less than $300 \mathrm{~Pa}$, within the clinically acceptable range.

\section{In Vitro Hydraulic Performance}

Hydraulic performance of a surface-modified viscous impeller pump (2-mm surface vanes and with cage present) was tested in the mock circulatory loop shown in Figure 4, $A$. The impeller induced a pressure increase of 0 to $20 \mathrm{~mm} \mathrm{Hg}$ and flow rates of 0 to $5 \mathrm{~L} / \mathrm{min}$ at rotational speeds of 0 to $7 \mathrm{~K} \mathrm{rpm}$ (Figure $4, B$ ). No reduction in flow rate was observed at $0 \mathrm{rpm}$, and no gross cavitation was observed at $7 \mathrm{~K} \mathrm{rpm}$.
TABLE 1. Transition from flow stabilizer to pump with increased rotation

\begin{tabular}{lccc}
\hline \multicolumn{1}{c}{ RPM } & $\begin{array}{c}\text { Pressure increase } \\
(\mathbf{m m ~ H g})\end{array}$ & $\begin{array}{c}\text { Maximum wall } \\
\text { shear (Pa)* }\end{array}$ & $\begin{array}{c}\text { Power } \\
\text { loss (watts) }\end{array}$ \\
\hline No impeller & -0.43 & 1.4 & 0.25 \\
0 & -0.05 & 7.6 & 0.03 \\
500 & -0.05 & 15.6 & 0.03 \\
1000 & -0.04 & 29.3 & 0.02 \\
2500 & 0.02 & 98.7 & -0.01 \\
5000 & 0.03 & 130.8 & -0.02 \\
\hline
\end{tabular}

$R P M$, Revolutions per minute; $P a$, pascal. *Location of maximum wall shear stress i on the TCPC vessel wall for case with no impeller and on impeller surface for cases with impeller.

Performance curves are characteristically flat, signifying consistent low-pressure performance over a wide range of flow conditions. At high pump speeds, the device is capable of generating pressure increase that is greater than the 2 to $5 \mathrm{~mm} \mathrm{Hg}$ optimum range because of the presence of surface vanes. Lower pressure increase values can be obtained at lower pump speeds or by using a more smoothly surfaced impeller (lower profile surface vanes). The operational speed of the pump and extent of surface vane expression may vary depending on the clinical conditions in which the pump is applied. The use of surface vanes to modify impeller performance must be balanced with acceptable shear rates at defined operational specifications.

\section{DISCUSSION}

Fontan Failure Is Not the Same as Heart Failure

Patients undergoing the Fontan procedure are at high risk of regression from compensated to uncompensated Fontan physiology not only at the time of repair but also as they age. ${ }^{5}$ This risk is, in part, a reflection of their initial staging operations in which a shunt was used. ${ }^{2}$ The increasing number of survivors with Fontan physiology constitutes an emerging public health concern. ${ }^{14}$ Although children and adults with "Fontan failure" exhibit classic features of congestive heart failure (increased tissue/organ water, decreased tissue/organ perfusion), the underlying cause is not primary myocardial dysfunction. The ventricular (diastolic) dysfunction observed in patients undergoing the Fontan procedure may be to a large extent attributable to chronically reduced filling. ${ }^{15-17}$

Treatment options for this group are limited and less than ideal. For example, diuretic therapy may alleviate symptoms of increased tissue water, but at the expense of circulating blood volume; ideal therapy would be to simply reduce systemic venous pressure alone. Similarly, inotrope therapy will improve cardiac output inefficiently in an underfilled ventricle; ideal therapy would be to simply increase ventricular filling. Cavopulmonary assist can ideally and concurrently address both of these circumstances. 


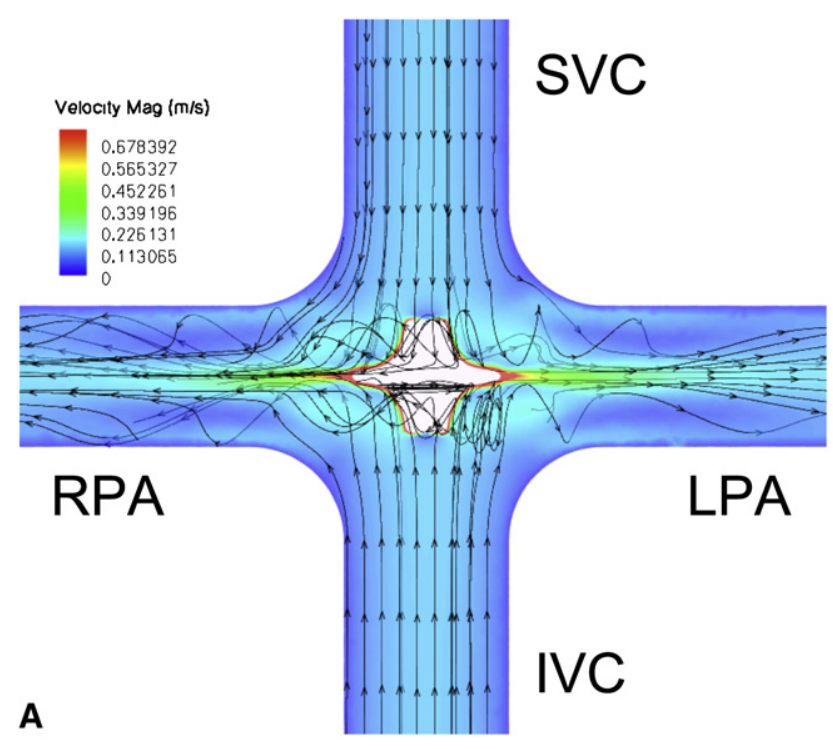

Scalar Stress (pa)

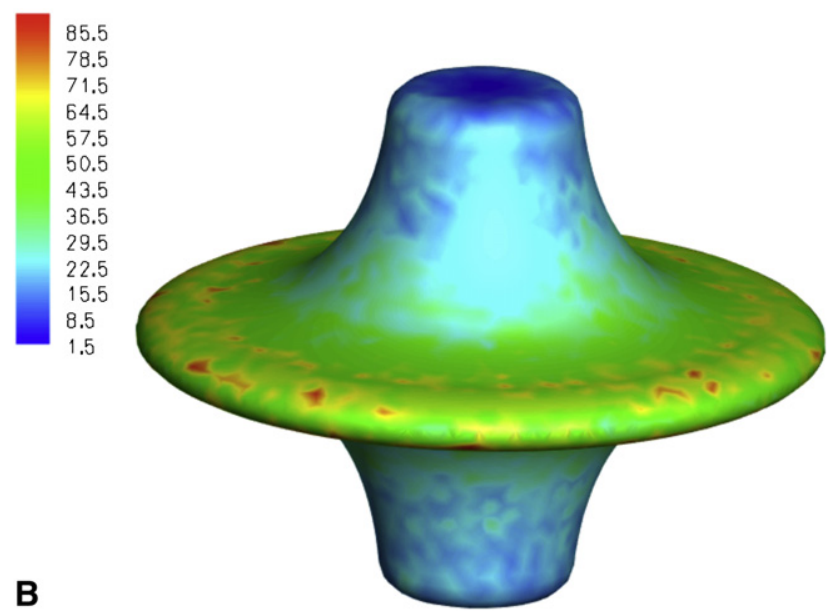

FIGURE 3. Predicted scalar stress (Pa) (A) and fluid streamlines as colored by total pressure $(\mathrm{Pa})(\mathrm{B})$ for a smooth surface viscous impeller pump rotating at $5000 \mathrm{rpm}$. $S V C$, Superior vena cava; $I V C$, inferior vena cava, $L P A$, left pulmonary artery; $R P A$, right pulmonary artery.

\section{A Pump "Primer" Rather Than a Primary Pump}

In a reversal of assumptions, it can be stated that other segments of the circulation are failing the Fontan heart. Thus (assuming normal systolic function), the target for therapeutic support of the Fontan circulation is the cavopulmonary circulation, rather than the systemic circulation. A cavopulmonary assist device would serve as a low input "primer" for the primary pump (systemic single ventricle), rather than as a primary pump per se. A modest increase in downstream ventricular filling $(2-5 \mathrm{~mm} \mathrm{Hg})$ will improve myocardial performance and cardiac output. Simultaneously, reduction in upstream systemic venous pressure will markedly benefit the systemic and mesenteric venous circulation and interstitium. This is identical to the essential function of the right ventricle in a normal biventricular circulation. ${ }^{18}$
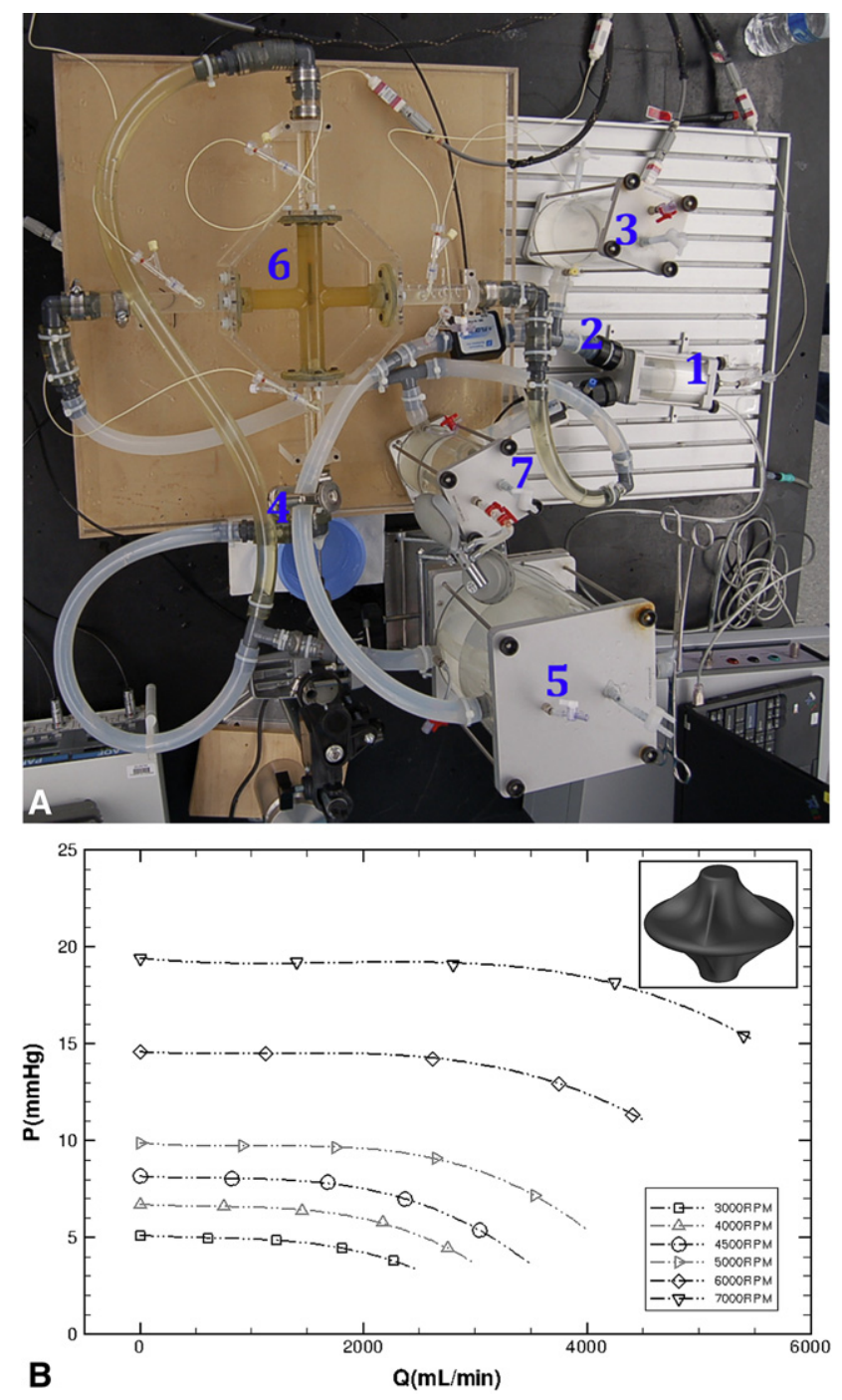

FIGURE 4. Prototype hydraulic performance. A, In vitro mock circulatory loop consisting of the following elements: (1) single ventricle, (2) aorta, (3) arterial compliance, (4) systemic vascular resistance, (5) venous compliance, (6) Fontan junction with viscous impeller pump, and (7) pulmonary compliance. B, Experimental hydraulic performance for a vaned impeller with cage present. $P$, Pressure millimeters of mercury; $Q$, flow milliliters/ minute.

\section{Unique Pump Requirements}

Mechanical cavopulmonary assist within the TCPC presents unique anatomic and physiologic challenges that are markedly dissimilar to any other mechanical circulatory support application. Flow must be augmented in a highly complex 3- or 4-axis geometry in which incoming and outgoing flow are perpendicularly opposed. The pump will provide partial support in a location where no ventricle will recover to assume function of the pump; it is not a ventricular assist device. Ambient hydrostatic pressure in the TCPC is low (10-12 mm $\mathrm{Hg}$ ), increasing concern for flow stasis and thrombogenicity. In addition, the desired flow is high-volume, low-pressure 

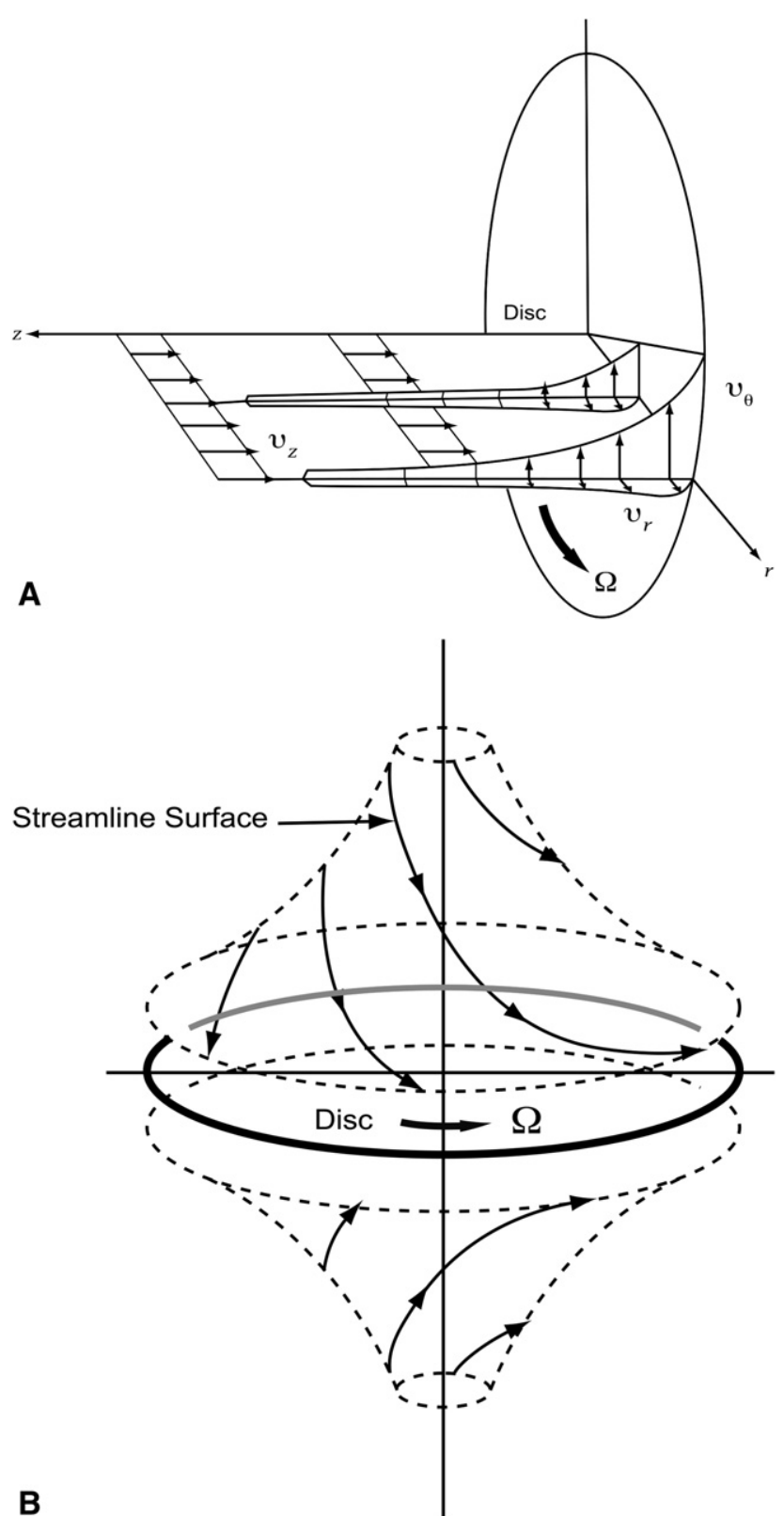

FIGURE 5. Von Kármán viscous pump. A, Flow patterns induced by actuator disk rotation. B, Flow streamlines depicted on 2 sides of an actuator disk.

flow. A gentle pressure step-up of 2 to $5 \mathrm{~mm} \mathrm{Hg}$ may be ideal. In certain cases, higher pressure may be undesirable or detrimental. In other instances, higher pressure may be necessary against increased pressure head (eg, pulmonary hypertension). Because there is no volume reservoir for the pump inlet to draw from, risk of vein collapse and cavitation caused by inlet suction is high. Thus, a high degree of fluid slip (low preload dependence) is preferable. Similarly, downstream flow must remain low pressure to avoid perfusion lung injury (similarly, a high degree of fluid slip is preferable). Notably, there is no natural barrier (eg, a valve) present within the
TCPC pathway to prevent recirculation around the pump body. For a fixed-diameter, small percutaneous device positioned within the large diameter central veins, recirculation must be addressed. Finally, and perhaps most important, it is critical that the cavopulmonary pathways remain unobstructed during pump deployment, as pump support is weaned to no net contribution before removal, when the pump is shut off, in the event of rotation failure, and after the pump is withdrawn.

\section{Limitations of Microaxial Flow Pumps}

At first thought, adapting a traditional axial flow design is an appealing solution to this problem. A computational model of an axial flow pump intended for use in the IVC has been reported in which blade geometry and rotational speed were simply modified to operate in the low-pressure venous circulation. ${ }^{19}$ On the contrary, in our experience, microaxial pumps have critical limitations. Most important, the pump itself, or combination of pump, housing, and barrier to recirculation, is obstructive to flow. Once in place, the pump cannot be simply shut off. In the event of failure or shutdown, the venous pressure necessary to maintain cardiac output would be untenable in an environment in which only 2 to $5 \mathrm{~mm} \mathrm{Hg}$ obstruction has grave consequences. For cavopulmonary assist, the ability to wean support to very low levels - without any degree of obstruction-is critical. Second, the inlet pressure of a microaxial pump is in an unacceptable negative range, portending suction collapse of the vena cava. The smaller the diameter of a fixed-radius microaxial pump, the greater the rotational speed required to transfer equivalent volume and the greater the risk for inefficient recirculation. A truly percutaneous microaxial device, at perhaps less than $10 \%$ of vena caval cross-sectional area, must greatly accelerate flow to deliver equivalent effective flow. This increases risk for suction, cavitation, and blood component activation. Third, because of size (anything $>4 \mathrm{~mm}$ in diameter in adults), placement and removal require open surgery and cardiopulmonary bypass. Fourth, a microaxial device requires a contained housing, inducer, and diffuser, which increase complexity, synthetic material burden, and thrombogenicity risk. Fifth, microaxial pump flow is unidirectional: This mandates either the use of 2 separate pumps or undesirable surgical revision of the systemic venous pathways to prevent retrograde pressure elevation and backflow into the opposing vena cava. $^{20,21}$

\section{An Expandable, Multidirectional Rotary Pump}

Because of these limitations, we abandoned existing microaxial designs and considered a rotary pump that is both expandable and multidirectional. The ability to expand the radius of a peripherally inserted, centrally deployed intravascular pump would reduce rotational rates and provide a functional barrier to recirculation. ${ }^{22}$ The native, endothelial-lined vessel walls would serve as the conduit for 


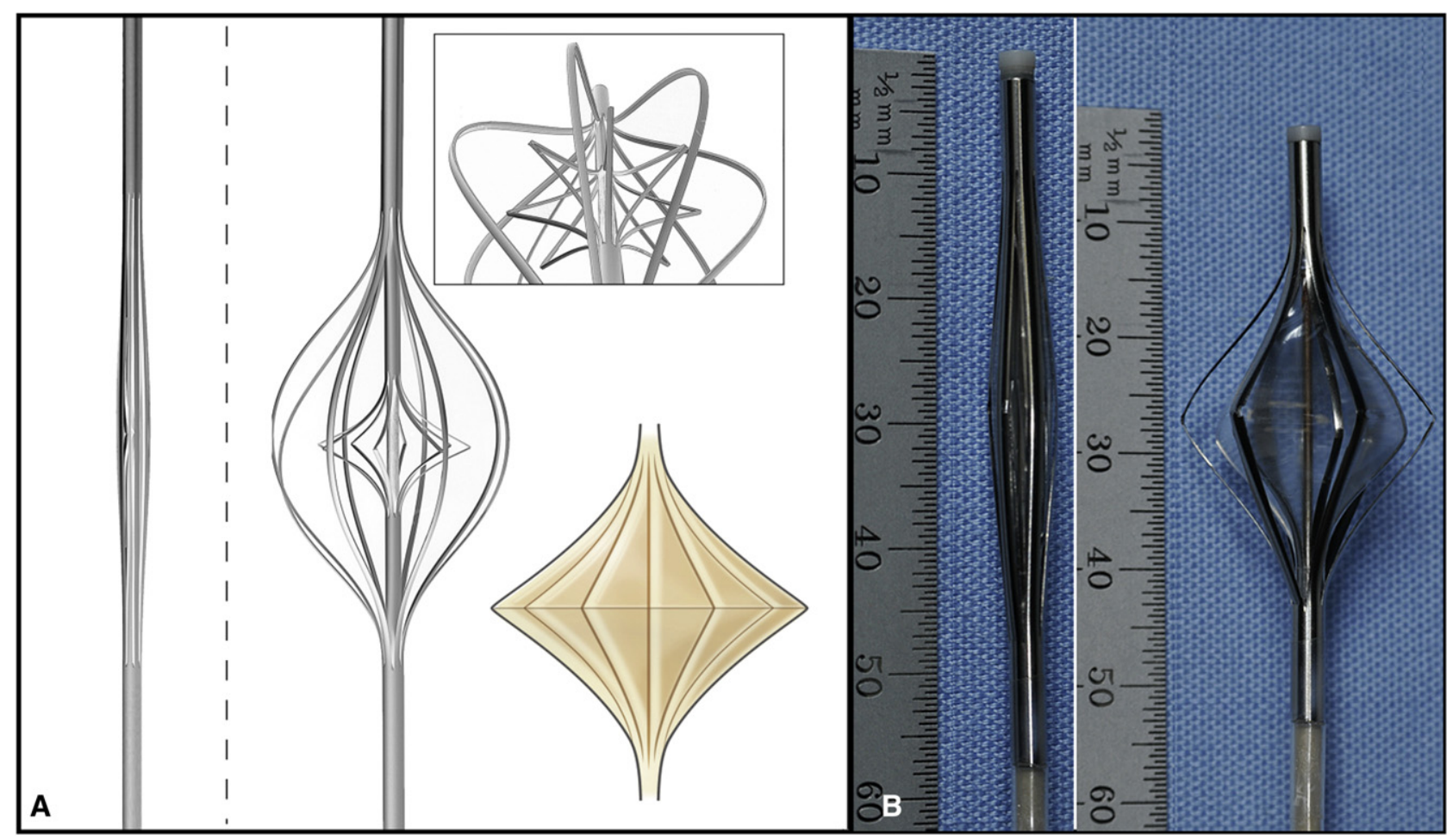

FIGURE 6. Expandable viscous impeller pump using "cage within a cage" concept. A, Large-scale model in collapsed configuration. B, Same model expanded by axial compression. The outer cage serves to center the impeller and protect the vessel wall. The inner cage, covered with an elastomer, forms the rotating impeller surface. The expanded to collapsed ratio is $500 \%$. C, Adult-scale functional prototype: closed diameter $8 \mathrm{~F}, 2.86 \mathrm{~mm}$; open diameter $22-\mathrm{mm}$ outer cage.

flow. Advanced numeric modeling has shown that a fixed central diverting body at the TCPC intersection will beneficially split incoming TCPC flow toward the outlets, minimizing turbulent energy loss. ${ }^{23}$ By combining passive flow optimization with the need for an expandable impeller, we had the insight to apply rotational energy to an expandable, central impeller suspended in the midst of the TCPC, independent of the vessel walls.

\section{von Kármán Viscous Pump}

Consider a large, flat disk rotated in fluid. ${ }^{6}$ The no-slip condition requires that fluid at the surface of the disk rotate with the same velocity (Figure 5, A). Viscous effects diffuse away from the disk and induce rotation. However, there is no pressure gradient in the radial direction to balance the centrifugal force. Once fluid has been accelerated by the plate, it is also flung out in a radial direction. Conservation requires that the outwardly displaced fluid is replaced by axial inflow from quiescent fluid far away from the disk. Thus, fluid is pumped from the far axial stream (SVC above; IVC below) toward the disk, where viscous forces induce a swirl and the resulting centrifugal effect produces radial outflow (Figure $5, B){ }^{7}$ On the basis of this fundamental fluid dynamic principal, a single impeller will stabilize and augment TCPC flow in 4 axes.
A viscous impeller pump is an exciting conceptual advance. The problem is dramatically simplified from a complex 2-pump solution with competing and colliding flows to a more advantageous, single impeller solution with multidirectional flow capability (3-way inverted "T"; 4-way "t"). The preferred TCPC pathway can be maintained, avoiding unnecessary surgical venous pathway revision that might be necessary with unidirectional devices. ${ }^{20,21}$ An impeller and protective centering cage, collapsed for percutaneous insertion, advanced to the intersection of the TCPC, opened, and rotated, will draw venous flow inward from opposed directions and propel blood radially outward in the pulmonary arteries, regardless of orientation (Figure 6).

\section{Broad Applicability of Cavopulmonary Assist}

Cavopulmonary assist is fully compatible with the existing staged paradigm and can be applied for temporary perioperative support after stage 2 or 3 repair alone. Cavopulmonary assist can also be applied late after repair for support of "failing Fontan" physiology in older children or adults. It can be implemented noninvasively in the catheterization laboratory or intensive care unit setting via percutaneous venous access and the Seldinger technique. Cavopulmonary assist may also enable a new paradigm of compressed or combined surgical staging: (1) stages 2 and 3 combined, or 1-stage Fontan 
conversion; (2) stages 1 and 2 combined (avoidance of shunt physiology) 24 ; (3) stages 1 to 3 combined (1-stage neonatal Fontan repair). This technology applies to both a 3-way "T" (stage 2 Glenn; hemi-Fontan) and 4-way " $t$ " (stage 3; TCPC). As a platform device, asymmetric impeller or surface (vane) modification can address differential inflow and outflow rates. In adults, the majority of complications of failing Fontan physiology arise from the IVC distribution, which accounts for $70 \%$ of total systemic venous return. Conversely, in neonates, upper-body venous return comprises $60 \%$ to $70 \%$ total venous flow, which is largely a reflection of cerebral blood flow ( 90\% SVC flow). ${ }^{25}$

\section{CONCLUSIONS}

This report describes a de novo pump concept and its anatomic and physiologic limitations and constraints. Much work remains to carry this concept to clinical implementation. A limitation of this preliminary analysis is that the impeller tested was a rigid rather than an expandable prototype. Furthermore, the device was scaled to adult dimensions; performance at dimensions suitable for infants and neonates remains to be determined. Future investigation will include reduced scale analyses, hydraulic optimization, and hemolysis and thrombogenicity assessment. Shear stress and hemolysis are not expected to be problems given the lower rotation requirements of this device in comparison with commercially available microaxial devices. A viscous impeller pump makes mechanical circulatory support of a univentricular Fontan circulation a more realistic consideration. Proven safe and effective, a viscous impeller pump will greatly improve therapy for individuals with singleventricle heart disease.

\section{References}

1. Ashburn DA, McCrindle BW, Tchervenkov CI, Jacobs ML, Lofland GK, Bove EL, et al. Outcomes after the Norwood operation in neonates with critical aortic valve stenosis or aortic valve atresia. J Thorac Cardiovasc Surg. 2003; 125:1070-82.

2. Khambadkone S, Li J, de Leval MR, Cullen S, Deanfield JE, Redington AN. Basal pulmonary vascular resistance and nitric oxide responsiveness late after Fontan-type operation. Circulation. 2003;107:3204-8.

3. Rodefeld MD, Boyd JH, LaLone BJ, Bezrucko AJ, Potter AW, Brown JW. Cavopulmonary assist: circulatory support for the univentricular Fontan circulation. Ann Thorac Surg. 2003;76:1911-6.

4. Rodefeld MD, Boyd JH, Myers CD, Presson RG, Wagner WW, Brown JW. Cavopulmonary assist in the neonate: an alternative strategy for single-ventricle palliation. J Thorac Cardiovasc Surg. 2004;127:705-11.

5. deLeval M. The Fontan circulation: what have we learned? What to expect? Pediatric Cardiol. 1998;19:316-20.

6. von Kármán T, Agnew Z. Math Mech. 1921;1:233.

7. Panton RL. Incompressible flow. 2nd ed. Malden, MA: Wiley InterScience; 1996:294.

8. Pantalos G. Use of computer and in vitro modeling techniques during the development of pediatric circulatory support devices. NHLBI Pediatric Assist Device Contractor's Meeting: Pediatric modeling techniques working group. ASAIO J. 2009;55:3-5.

9. Migliavacca F, Dubini G, Bove EL, de Leval MR. Computational fluid dynamics simulations in realistic 3-D geometries of the total cavopulmonary anastomosis: the influence of the inferior caval anastomosis. J Biomech Eng. 2003;125:805-13.
10. Pekkan K, Zelicourt DD, Ge L, Sotiropoulos F, Frakes D, Fogel MA, et al. Physics-driven CFD modeling of complex anatomical cardiovascular flows-a TCPC case study. Ann Biomed Eng. 2005;33:284-300.

11. Bludszuweit $\mathrm{C}$. Three-dimensional numerical prediction of stress loading of blood particles in a centrifugal pump. Artif Organs. 1995;19:590-6.

12. Apel J, Paul R, Klaus S, Siess T, Reul H. Assessment of hemolysis related quantities in a microaxial blood pump by computational fluid dynamics. Artif Organs. 2001;25:341-7.

13. Bludszuweit C. Model for general mechanical blood damage prediction. Artif Organs. 1995; 19:583-9.

14. Williams R, Pearson GD, Barst RJ, Child JS, del Nido P, Gersony WM, et al Report of the National Heart, Lung, and Blood Institute Working Group on Research in Adult Congenital Heart Disease. J Am Coll Cardiol. 2006;47:701-7.

15. Eicken A, Petzuch K, Marek J, Vogel M, Hagler A, Vogt M, et al. Characteristics of Doppler myocardial echocardiography in patients with tricuspid atresia after total cavopulmonary connection with preserved systolic ventricular function. Int J Cardiol. 2007;116:212-8.

16. Anderson PA, Sleeper LA, Mahony L, Colan SD, Atz AM, Breitbart RE, et al. Contemporary outcomes after the Fontan procedure. J Am Coll Cardiol. 2008; 52:85-98.

17. Backer CL. The Fontan procedure. Our odyssey continues (editorial). J Am Coll Cardiol. 2008;52:114-6.

18. Furey SA, Zieske HA, Levy MN. The essential function of the right ventricle. Am Heart J. 1984;107:404-10.

19. Wang R, Lacour-Gayet F, Lanning CJ, Rech BA, Kilfoil PJ, Hertzberg J, et al. Initial experience with the development and numerical and in vitro studies of a novel low-pressure artificial right ventricle for pediatric Fontan patients. ASAIO J. 2006;52:682-92

20. Lacour-Gayet FG, Wang R, Lanning CJ, Goldberg SP, Rech BA, Shandas R. Experimental study of an artificial right ventricle for failing Fontan: in vitro and computational evaluation (abstract). The Society of Thoracic Surgeons, 45th Annual Meeting, Jan 27-30, 2009.

21. Marsden AL, Bernstein AJ, Reddy VM, Shadden SC, Spilker RL, Chan FP, et al. Evaluation of a novel Y-shaped extracardiac Fontan Baffle using computational fluid dynamics. J Thorac Cardiovasc Surg. 2009;137:394-403.

22. Throckmorton AL, Ballman KK, Myers CD, Frankel SH, Brown JW, Rodefeld MD. Performance of a 3-bladed propeller pump to provide cavopulmonary assist in the failing Fontan circulation. Ann Thorac Surg. 2008;86: 1343-7.

23. Soerensen DD, Pekkan K, de Zelicourt D, Sharma S, Kanter K, Fogel M, et al Introduction of a new optimized total cavopulmonary connection. Ann Thorac Surg. 2007;83:2182-80.

24. Honjo O, Merklinger SL, Poe JB, Guerguerian AM, Alghamdi AA, Takatani S, et al. Mechanical cavopulmonary assist maintains pulmonary and cerebral blood flow in a piglet model of a bidirectional cavopulmonary shunt with high pulmonary vascular resistance. J Thorac Cardiovasc Surg. 2009;137:355-61.

25. Fogel M, Durning S, Wernovsky G, Pollock AN, Gaynor JW, Nicolson S. Brain versus lung: hierarchy of feedback loops in single-ventricle patients with superior cavopulmonary connection. Circulation. 2004;suppl II:II147-52.

\section{Discussion}

Dr Glen Van Arsdell (Toronto, Ontario, Canada). With this presentation, Dr Rodefeld and his colleagues have introduced an important conceptual leap around the ideas for mechanical assist in single-ventricle palliation. This is the first time one can actually envision one of the holy grails for single-ventricle surgery, ie, 1-stage neonatal Fontan with outcomes that match neonatal biventricular repair. Except for incremental improvements in singleventricle surgery, it appears to me that we have reached a ceiling. Now we can see beyond that ceiling, at least conceptually. I have 3 questions for you. One, in your model, it appears that the study is based on a rigid wall concept. In humans, there is some rigidity and some flexibility in a standard Fontan setup. Can you talk with us about how you envision dealing with this conceptually? I know you mentioned the cage, but one of the issues with the microaxial devices is wall trauma and cavitation. 
Dr Rodefeld. What you are seeing is the first iteration of the pump concept, and it is demonstrated in an idealized rigid conduit. There are ways to model a compliant vessel, either by using a computational technique or by in vitro modeling, and certainly those would be done with additional studies, and very eloquent modeling can account for vessel compliance in this situation.

Dr Van Arsdell. So do you have a sense of the "suction" effects on the walls in this device as compared to a microaxial device?

Dr Rodefeld. I don't have any hard data on it. This type of pump is a slippery pump, it is a weak pump, and it is the reason why you don't see it used in a lot of industrial applications. But my sense is that the negative pressure generation is going to be much less of a problem than what you would see with a fixed radius, microaxial flow pump that has to spin at high speed.

Dr Van Arsdell. Second, many of the patients that may potentially benefit from this are our current failing Fontan population, which is primarily an atriopulmonary connection type of arrangement. Would you envision this usage in that connection or would it require a conversion to the present extracardiac Fontan arrangement?

Dr Rodefeld. This particular device would not apply necessarily for an atriopulmonary type of construction. The field has moved away from that, but obviously the older failing Fontan patients that we are dealing with presently had the prior atriopulmonary type of reconstruction. I think at this point I would advocate conversion to a total cavopulmonary connection, which, as you know, gives the benefit of 2 to $5 \mathrm{~mm} \mathrm{Hg}$ pressure streamlining, which actually supports this pump impeller concept as well, and then if they need additional temporary support, this pump could provide that.

Dr Van Arsdell. It would seem to me one of the advantages of this is that you might raise the margin of safety around Fontan conversion to a more modern Fontan.

My final question: One of the most remarkable things in this presentation to me is the level of efficiency of flow seen with the static implantation of a disk alone. Are you looking at or should we be looking at a biologically compatible disk that could be suspended magnetically within the current Fontan arrangement, ie, implanted at the time of Fontan or that could be implanted percutaneously later?

Dr Rodefeld. This could be potentially placed as a static device percutaneously, similar to a venocaval filter type of deployment or retrieval strategy. There are other groups that have talked about a static device in the cavopulmonary connection. Most important would be the Georgia Tech group and Ajit Yoganthan have reported on a flow splitting device that would optimize streamlining through the cavopulmonary connection. This could be considered as a static device, but the pressure reduction is fairly small. So whether that would have a clinical impact or not, I am not sure at this point.

Dr Van Arsdell. Congratulations on this excellent manuscript. Dr Rodefeld. Thank you. 